\title{
Biological activity of the thrombospondin epidermal growth factor domain from banana shrimp (Fenneropenaeus merguiensis)
}

\author{
Monwadee Wonglapsuwan $^{\mathrm{a}, \mathrm{b}}$, Natthaporn Khwanmuang ${ }^{\mathrm{a}}$, Panchalika Deachamaga, \\ Wilaiwan Chotigeat ${ }^{\mathrm{a}, \mathrm{b}, *}$ \\ a Department of Molecular Biotechnology and Bioinformatics, Faculty of Science, Prince of Songkla \\ University, Songkhla 90112 Thailand \\ b Center for Genomics and Bioinformatics Research, Faculty of Science, Prince of Songkla University, \\ Songkhla 90112 Thailand
}

*Corresponding author, e-mail: wilaiwan58@hotmail.com

Received 7 Sep 2019

Accepted 24 Jan 2020

\begin{abstract}
Human epidermal growth factor (EGF) plays a role in cell proliferation and has been used to promote wound healing in patients. However, its role in cancer development via the EGF receptor (EGFR) has recently been reported. Thrombospondin (TSP) from banana shrimp (Fenneropenaeus merguiensis) contains an EGF domain at its carboxy-terminal end though it shares only 30\% nucleotide sequence similarity with human EGF. It is challenging to investigate its biological activities. In this work, the EGF domain from banana shrimp TSP (TSP3-2) was cloned, and the recombinant His-TF-TSP3-2 was evaluated for its effects on proliferation, cytotoxicity and collagen production in a skin fibroblast cell line. Additionally, genotoxicity was determined using a hypoxanthine-guanine phosphoribosyltransferase mutation assay. Fibroblast cell proliferation increased 1.2-1.4 fold after treatment with 0.5-15 $\mu \mathrm{g} / \mathrm{mL}$ His-TF-TSP3-2. The migration rate after $48 \mathrm{~h}$ of the His-TF-TSP3-2-treated cells (at $0.5 \mu \mathrm{g} / \mathrm{mL}$ ) in a scratch assay was greater than that of the control. The collagen production was 1.7- and 1.2-fold increased after treatment with His-TF-TSP3-2 at 0.5 and $1.0 \mu \mathrm{g} / \mathrm{mL}$, respectively. Low toxicity to the genome and no significant mutant frequency was found in the His-TF-TSP3-2-treated cells. Further investigations are required to confirm a potential use for this protein.
\end{abstract}

KEYWORDS: thrombospondin (TSP), proliferation, growth factor, collagen

\section{INTRODUCTION}

Extracellular matrix proteins are known to maintain tissue and organ structures. They have a range of functions including acting as signal modulators involved in cell adhesion, proliferation, migration, and differentiation [1-3]. Thrombospondins are glycoproteins in the extracellular matrix and have been reported to have various functions in humans [2]. For example, the thrombospondins TSP1 and TSP2 are potent endogenous inhibitors of angiogenesis [3]; TSP1 can cause an imbalance in the host immune response that ultimately leads to reduced phagocytic function [4] and potently inhibits fibroblast migration [5]. The human TSPs, including TSP-1, TSP2, TSP-3, TSP-4, and TSP5/COMP, have been classified [6]. The general structure of human TSPs consists of repeated chitinbinding domains, an EGF-like domain, repeated thrombospondin-type domains and a C-terminal do- main [2] while Penaeus monodon TSP (PmTSP) contains 4 chitin-binding type II domains, an EGFlike domain, 8 thrombospondin type III domains and a thrombospondin C-terminal domain. Additionally, Marsupenaeus japonicas TSP (MjTSP) has 5 chitin-binding type II domains, 6 EGF-like domains, 8 thrombospondin type III domains and a thrombospondin C-terminal domain [7]. In humans, TSP transcripts have been found in organs such as the intestine, ovary, and heart. Each human TSP exhibits high expression in a specific organ. For example, TSP1, TSP2, TSP3, TSP4, and TSP5 show the highest expression in the lung, ovary, uterus, pituitary gland, and trachea, respectively [2]. Both PmTSP and MjTSP are TSP3 genes and are expressed at very high level in the ovary, intestine and heart $[7,8]$.

Some TSPs are localized to different cell types or structures within the same tissue. The expression of TSPs changes in response to physiological or pathological changes; for example, TSP1 is found at 
high level during ischemia. Moreover, TSP1 appears to exert a protective effect in myocardial infarction (MI) patients, in whom the high level is present [9]. TSP1 plays a role in collagen homeostasis through interactions with matrix metalloproteinases and directly affects collagen fibril formation [10]. TSP4 has been reported to protect against heart disease after MI injury or protein misfolding [11]. TSP4 expression is induced in the heart and vasculature under pathological conditions including myocardial infarction, myocardial pressure overload, and hypertension. TSP4 is linked to remodeling processes, in which it may affect extracellular matrix protein organization [12]. TSP5 has been reported to support chondrocyte attachment through interactions with integrins [13].

In shrimp, only TSP3 has been reported, and it exhibits high expression in ovarian tissues. The structures of TSPs from P. monodon, Fenneropenaeus chinensis and $M$. japonicus show fewer EGF domains than human TSPs $[7,14,15]$. The corresponding biological activities have not been reported. Therefore, TSP from the ovarian tissue of banana shrimp (F. merguiensis) was cloned and characterized in this study.

Currently, increasing numbers of slow or nonwound healing patients are found among the aging and obese populations [16]. Bioactive compounds that enhance wound healing have been introduced such as EGF, growth hormone, and basic fibroblast growth factor [17-19]. The topical use of EGF was reported to increase epithelial cell proliferation and reduce the healing time of skin grafts, venous ulcers, and diabetic foot ulcers, and it was found that epidermal growth factor receptor (EGFR) expression was increased [20]. EGFR was observed to play a critical role in oncogenesis and metastasis while EGFRi (EGFR inhibitor) is a promising target for the treatment of metastatic cancers. However, EGFR inhibition showed significant cutaneous side effects and limitations to long-term use [16,21]. Therefore, an alternative EGF without a role in cancer progression or a specific EGFRi that does not affect EGFR signaling in normal cellular function is needed. And the EGF domain of TSP has this required character. Thus, the EGF domain of TSP was cloned and evaluated for its biological activities such as wound healing, collagen production, and genotoxicity.

\section{MATERIALS AND METHODS}

\section{Banana shrimp TSP sequence analysis}

Total RNA was extracted from banana shrimp ovaries using the TRIzol reagent (Invitrogen, Carlsbad, CA, USA) following the manufacturer's protocol. To convert the RNA to cDNA, $400 \mathrm{ng}$ of total RNA was incubated with $200 \mathrm{ng}$ of random primers at $70{ }^{\circ} \mathrm{C}$ for $5 \mathrm{~min}$, followed by cooling on ice for $5 \mathrm{~min}$. This mixture was supplemented with $1 \times$ avian myeloblastosis virus (AMV) buffer, $1 \mathrm{mM}$ deoxynucleotide triphosphate, and $10 \mathrm{U}$ of AMV reverse transcriptase (Promega, Madison, USA) in a $25 \mu \mathrm{L}$ reaction mixture, followed by incubation at $48^{\circ} \mathrm{C}$ for $2 \mathrm{~h}$. Primers for full-length TSP were designed based on the GenBank database accession number FJ644567 as follows: forward primer, 5'CATATGATGGCGTTTCTTTCCGCAA- $3^{\prime}$, and reverse primer, 5 '-CTCGAGTTATTGGTTAGACTTGCCC- ${ }^{\prime}$. The obtained PCR fragment was cloned into the pGEM-T Easy vector (Promega, Madison, USA), and the nucleotide sequence was determined with bioinformatics tools. Phylogenetic analysis was analyzed by the neighbor joining (NJ) and maximum likelihood (ML) methods using MEGA software. Five hundred bootstrap replications were performed for the NJ trees to check the reproducibility of the results. TSP3-2 sequence alignment was performed by ClustalX2 and GeneDoc software. Then, the TSP3-2 fragment was cloned into the pGEM-T Easy plasmid. The primers for the TSP3-2 gene were as follows: forward primer, 5'-CATATGCA TTGTCCAGAGACCGATCCA- $3^{\prime}$, and reverse primer, 5'-ACAGTATCTAACCCAAGCCAAAAGCTT-3'.

\section{Preparation of banana shrimp TSP3-2 protein}

The shrimp TSP3-2 gene was cloned in frame with a histidine fusion system into the HindIII and NdeI restriction sites in the pCold vector (Takara, Tokyo, Japan) to obtain the pCold-TSP3-2 clone. In the first step, the TSP3-2 fragment and pCold vector were digested with the HindIII and NdeI restriction enzymes. The fragments were observed by a $1.2 \%$ agarose gel and then purified from the gel with the QIAquick PCR purification kit (QIAGEN, USA). TSP3-2 was subsequently ligated into the pCold vector at the HindIII and NdeI restriction sites. The whole ligation reaction was incubated with competent $E$. coli Top10F' cells. Colonies were grown overnight at $37^{\circ} \mathrm{C}$ on $100 \mu \mathrm{g} / \mathrm{mL}$ ampicillin plates. The plasmid DNA was extracted to select positive clones. The pCold-TSP3-2 clone was then 
transformed into E. coli BL21(DE3) cells.

The $E$. coli strain BL21(DE3) containing pColdTSP3-2 was grown overnight at $37^{\circ} \mathrm{C}$ in LB medium containing $100 \mu \mathrm{g} / \mathrm{mL}$ ampicillin. Pre-warmed medium $(300 \mathrm{~mL})$ was inoculated with $30 \mathrm{~mL}$ of an overnight culture and grown at $37^{\circ} \mathrm{C}$ with vigorous shaking until a log-phase OD was reached. Protein expression was induced with $1 \mathrm{mM}$ isopropyl- $\beta$-Dthiogalactopyranoside (IPTG), and the cells were further grown at $30^{\circ} \mathrm{C}$ for $16-18 \mathrm{~h}$. The bacterial cells were harvested by centrifugation $(4000 \times g$ for $20 \mathrm{~min}$ ), resuspended in lysis buffer $(50 \mathrm{mM}$ $\mathrm{NaH}_{2} \mathrm{PO}_{4}, \mathrm{pH} 8.0,300 \mathrm{mM} \mathrm{NaCl}, 6 \mathrm{M}$ urea and $5 \mathrm{mM}$ imidazole), lysed by sonication $(20 \times 10 \mathrm{~s}$, $200-300 \mathrm{~W}$ ), and centrifuged at $2000 \times \mathrm{g}$ for $20 \mathrm{~min}$ at $4{ }^{\circ} \mathrm{C}$. The cell debris and supernatant were examined by an SDS-PAGE gel with Coomassie brilliant blue staining.

The protein in the supernatant was further purified by elution from Ni-NTA resin connected to the AKTA prime chromatography system (GE Healthcare Bio-Sciences AB, Uppsala, Sweden). The column was equilibrated with $25 \mathrm{~mL}$ of $50 \mathrm{mM}$ phosphate buffer $\mathrm{pH} 7.4$ containing $300 \mathrm{mM} \mathrm{NaCl}$ and $10 \mathrm{mM}$ imidazole and washed with $300 \mathrm{~mL}$ of the same buffer at a flow rate of $5 \mathrm{~mL} / \mathrm{min}$. Then, $10 \mathrm{~mL}$ of the sample was injected and eluted with $30 \mathrm{~mL}$ of the same phosphate buffer but with $300 \mathrm{mM} \mathrm{NaCl}$ and $250 \mathrm{mM}$ imidazole. Ten fractions $(1 \mathrm{~mL})$ were subsequently collected from the flowthrough. The proteins in each fraction were detected by $12 \%$ SDSPAGE and Western blot analysis. To remove imidazole, the purified His-TSP3-2 protein was dialyzed in the same phosphate buffer with $300 \mathrm{mM} \mathrm{NaCl}$ for $24 \mathrm{~h}$. The purified proteins were kept at $-80^{\circ} \mathrm{C}$ until use.

\section{Thermal stability of HIS-TF-TSP3-2}

The thermal stability of His-TF-TSP3-2 was analyzed using Thermal shift assay methods [22]. The 5000X SYPRO orange fluorescent stain for protein was diluted 50-fold in $100 \mathrm{mM}$ HEPES with $150 \mathrm{mM} \mathrm{NaCl}$, $\mathrm{pH}$ 7.5. The reaction consisted of $5 \mathrm{X}$ SYPRO orange, $100 \mathrm{mM}$ buffer, $100 \mathrm{mM} \mathrm{NaCl}$, and $0.138 \mu \mathrm{g} / \mathrm{mL}$ His-TSP3-2 protein. Deionized water was added instead of the test protein in the control samples. The reactions were centrifuged for $1 \mathrm{~min}$ at 1000 rpm. The tubes were heated in a Mx3005P QPCR system from $25-90^{\circ} \mathrm{C}$ in increments of $0.5^{\circ} \mathrm{C}$. A graph of the relationship between temperature and the fluorescent signal was plotted and analyzed. The melting temperature was determined from the midpoint of the unfolding transition.

\section{Skin fibroblast proliferation and viability assay}

A human skin fibroblast cell line (Millipore's FibroGROTM Xeno-Free Human Foreskin Fibroblasts, USA) was cultured in Dulbecco's modified Eagle's medium (DMEM, Gibco, USA) containing 10\% fetal bovine serum (FBS, Gibco, USA) and antibiotics $(100 \mathrm{U} / \mathrm{mL}$ penicillin and $100 \mathrm{U} / \mathrm{mL}$ streptomycin, Gibco, USA) with $5 \% \mathrm{CO}_{2}$ at $37^{\circ} \mathrm{C}$. The media was changed every other day. When the cells reached confluence, they were harvested using $0.25 \%$ trypsin-EDTA (Gibco, USA), followed by the addition of fresh culture medium to generate a new single-cell suspension for further cell passaging.

Cytotoxicity was evaluated by the method described [23]. The skin fibroblast cell line was seeded at a concentration of $5 \times 10^{4}$ cells $/ \mathrm{mL}$ into a 96well plate in DMEM containing 10\% FBS. After $24 \mathrm{~h}$, the culture medium was replaced by fresh medium. His-TF-TSP3-2 at various concentrations (i.e., 0, 0.5, $1,5,7.5,10,15$, and $30 \mu \mathrm{g} / \mathrm{mL}$ ) in fresh medium was added to the culture medium. Cells without His-TF-TSP3-2 served as the negative control. After incubation for $24 \mathrm{~h}$, a 3-(4,5-dimethyl thiazolyl2-yl)-diphenyl-tetrazolium bromide (MTT) solution $(5 \mathrm{mg} / \mathrm{mL})$ assay was performed to evaluate cell activity. Briefly, the cells were treated with $100 \mu \mathrm{L}$ of fresh medium along with $50 \mu \mathrm{L}$ of MTT solution, followed by incubation at $37^{\circ} \mathrm{C}$ with $5 \% \mathrm{CO}_{2}$ for $4 \mathrm{~h}$. Thereafter, the medium containing MTT was removed, and $100 \mu \mathrm{L}$ of DMSO was added. The absorbance was determined with a microplate reader (Biohit 830, Helsinki, Finland) at a wavelength of $570 \mathrm{~nm}$. The percentage of cell proliferation was calculated and compared to the negative control.

\section{In vitro scratch assay}

The spreading and migration capabilities of human skin cells were assessed using a scratch assay (injury to the cell monolayer) that measured the expansion of a cell population on a surface as described [26, 27]. The skin fibroblast cell line was seeded at a concentration of $9 \times 10^{5}$ cells/mL in DMEM containing $10 \%$ FBS in a 6-well plate. Once a confluent monolayer was formed, a linear scratch was generated in the monolayer with a sterile pipette tip. Any cellular debris was removed and replaced with $2 \mathrm{~mL}$ of His-TF-TSP3-2 and His-TF at a concentration of $0.5 \mu \mathrm{g} / \mathrm{mL}$ in fresh medium, and DMEM without a sample served as a control. Microphotographs were taken at $10 \times$ magnification using an Olympus IX73 inverted microscope (Bangkok, Thailand) on day 0 . Then, the plates were incubated with $5 \% \mathrm{CO}_{2}$ at 
$37^{\circ} \mathrm{C}$, and photographs were taken at 24,36 , and $48 \mathrm{~h}$ after incubation. The images acquired for each sample were further analyzed quantitatively using computing software (ImageJ 1.42q/Java 1.6.0 10). By comparing the images from $0-24,36$, and $48 \mathrm{~h}$, the distance of each scratch closure was determined and the percent migration rate was calculated. The average values for the left scratch and right scratch were determined separately. The percent migration was calculated for the left scratch and then the right scratch. Samples were examined in triplicate. The percentage of migration obtained from three wells was averaged and recorded.

$$
\text { Migration rate }=\frac{\mathrm{DAS}_{0}-\mathrm{DAS}_{\mathrm{ep}}}{\mathrm{DAS}_{0}}
$$

where $\mathrm{DAS}_{0}$ and $\mathrm{DAS}_{\text {ep }}$ are the average distance across the scratch (day 0) and across the scratch (experimental periods), respectively.

\section{Determination of soluble collagen production}

Soluble collagen was quantified by the method described [23]. The BJ human skin fibroblast cell line was seeded at an initial concentration of $2 \times 10^{5}$ cells $/ \mathrm{mL}$ in 96-well plates in DMEM supplemented with $10 \%$ FBS. After $24 \mathrm{~h}$, the culture medium was replaced with fresh DMEM plus 10\% FBS, and His-TF-TSP3-2 was added to the culture medium at concentrations of 0.5 and $1 \mu \mathrm{g} / \mathrm{mL}$ in fresh medium. After incubation for $24 \mathrm{~h}$, the supernatants were collected. Then, the total amount of soluble collagen was assayed using the Sorcol collagen assay kit (Biocolor Ltd., Carrickfergus, UK). Briefly, $100 \mu \mathrm{L}$ of the experimental cell supernatant was mixed gently with $1 \mathrm{~mL}$ of dye solution at room temperature for $30 \mathrm{~min}$. Thereafter, the samples were centrifuged at $15000 \times g$ for $10 \mathrm{~min}$ to form a collagen pellet. The supernatant was removed, and the soluble collagen was dissolved in $1 \mathrm{~mL}$ of alkali reagent. The resultant solutions with the alkali reagent were transferred to a 96-well plate, and measurements were performed with a microplate reader at a wavelength of $540 \mathrm{~nm}$. The amount of collagen was calculated based on a soluble collagen standard curve. DMEM supplemented with 10\% FBS without His-TSP protein was used as a negative control.

\section{Determination of TSP3-2 protein genotoxicity}

CHO cells $\left(1 \times 10^{5}\right.$ cells $)$ were seeded per well in a 48 well plate and incubated for 36-40 h. The cells were exposed to a concentration of 0.5 or $1 \mu \mathrm{g} / \mathrm{mL}$ of His-TF-TSP3-2 protein for 24, 48, or $72 \mathrm{~h}$ at $37^{\circ} \mathrm{C}$, after which the medium containing the His-TF-TSP3-2 protein was removed. The 1-methyl-3-nitro-1-nitrosoguanidine (MNNG) (Tokyo Chemical Industry Co., Ltd, Japan) and 7,12-dimethylbenz(a)anthracene (DMBA) (Sigma Aldrich, US) mutagens were used as positive controls while PBS and DMSO were used as negative controls. The cells were cultured in DMEM for 8 days. Mutant cell selection was performed by treatment with $5 \mu \mathrm{g} / \mathrm{mL}$ 6-thioguanine (6-TG) (Sigma Aldrich, US) in DMEM. After 10 days of growth, the cells were stained and counted to determine the mutant frequency.

\section{Statistical analysis}

Three separate samples were used for all the experiments in this study. The mean of three samples was calculated. The results were statistically compared between groups using the independent samples $t$ test via SPSS software at a $95 \%$ confidence level $(p<0.05)$.

\section{RESULTS}

\section{FmTSP sequence analysis and preparation of HIS-TF-TSP3-2}

Full-length FmTSP was amplified based on information from the GenBank database. The nucleotide sequence of the obtained PCR fragment was determined and analyzed. The full-length TSP sequence contained 2769 bp encoding 922 amino acids. FmTSP was found to consist of 3 chitinbinding repeats, an epidermal growth factor domain (TSP3-2), 8 thrombospondin-3 domains and a thrombospondin C-terminal domain (Fig. 1ab). Phylogenetic analysis of the TSP family was performed, and the results revealed that TSPs from shrimp are closely related to each other. Moreover, shrimp FmTSP was closer to TSP3 and TSP4 than TSP1 and TSP2 (Fig. 1c). The epidermal growth factor domain of FmTSP was defined as TSP3-2, and it was cloned to characterize its activity for potential applications. TSP3-2 was $462 \mathrm{bp}$ in length and translated into 154 amino acids (aa); when the protein sequence of TSP3-2 was compared with EGF proteins from other organisms, it displayed 90\% identity with $F$. chinensis and $P$. monodon (Fig. 1d). The TSP3-2 nucleotide sequence showed only 30\% similarity to human EGF, and the TSP3-2 domain contained 13 phosphorylation sites including 9 serine, 1 threonine, 2 tyrosine and 6 cysteine residues, which are characteristic of epidermal growth factor 
(a)

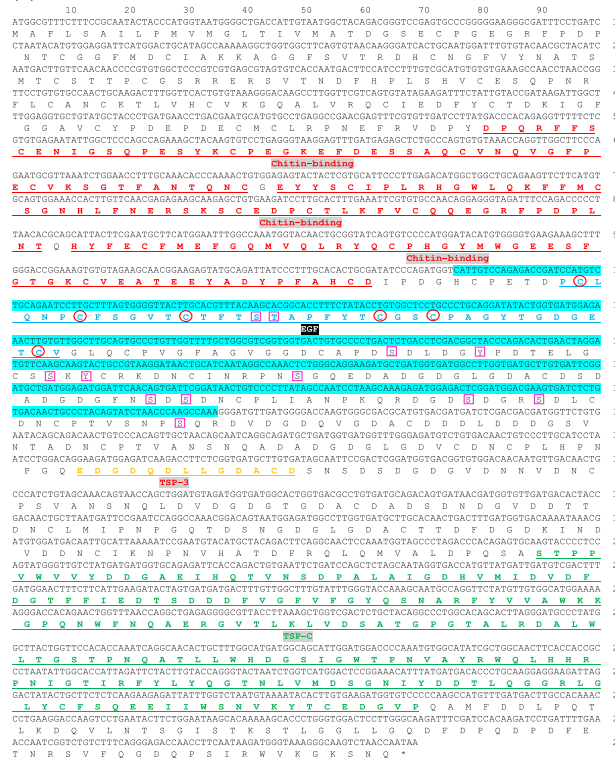

(b)
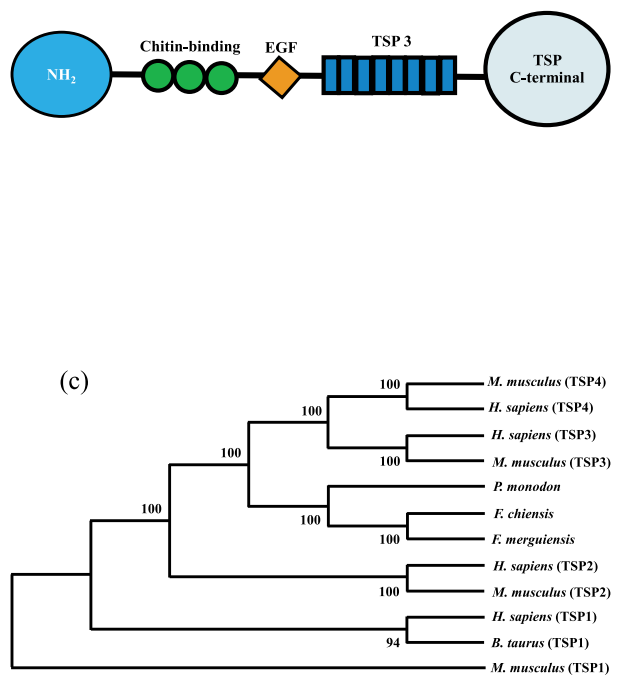

(d)

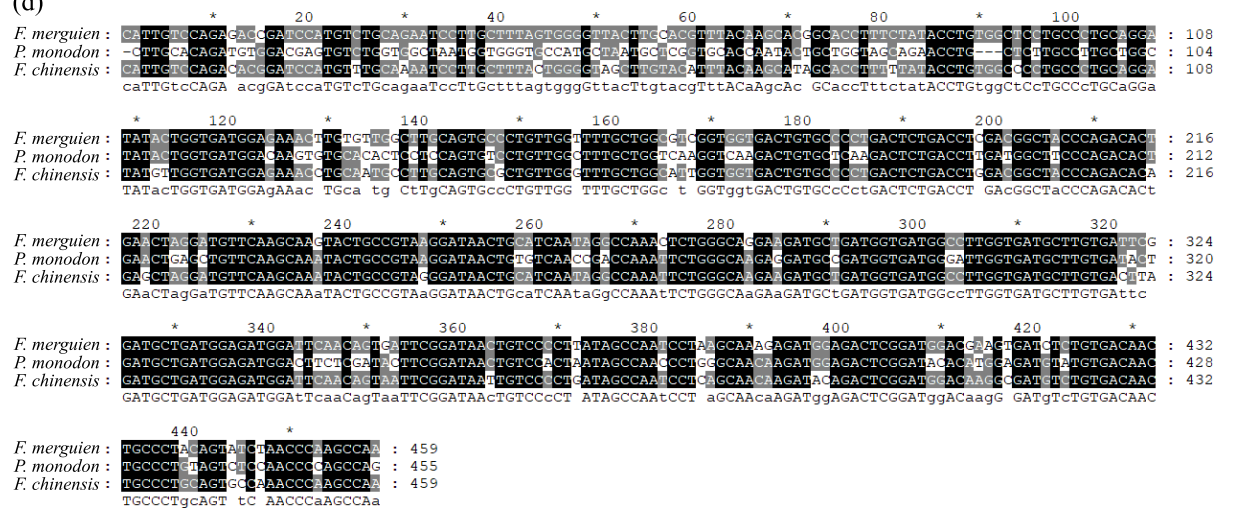

Fig. 1 Nucleotide and deduced amino acid sequences of the FmTSP. (a) The nucleotide and amino acid sequences. An asterisk denotes the termination codon. The chitin-binding repeats are underlined in red. The epidermal growth factor domain, thrombospondin type III repeats (TSP-3) and thrombospondin C-terminal domain (TSP-C) are indicated in blue, yellow, and green letters, respectively. The blue highlight indicates the TSP3-2 domain. The kinase phosphorylation sites including 9 serine, a threonine, and 2 tyrosine residues are marked with rectangles. Cysteines are marked with circles. (b) The cartoon summarizes the structure of FmTSP. (c) Phylogenetic analysis (using MEGA X) of the FmTSP gene and the TSPs from other shrimp, animals and human with sequences from GenBank. M. musculus (TSP4), accession no. NM011582.3; H. sapiens (TSP4), accession no. NM003248.6; H. sapiens (TSP3), accession no. NM007112.5; M. musculus (TSP3), accession no. NM013691.3; P. monodon, accession no. JX413010.2; F. chiensis, accession no. DQ091254.1; F. merguiensis, accession no. FJ644567.1; H. sapiens (TSP2), accession no. NM003247.4, M. musculus (TSP2), accession no. NM011581.3; M. musculus (TSP1), accession no. NM011580.4; H. sapiens (TSP1), accession no. NM003246.4; and B. taurus (TSP1), accession no. NM174196.1. (d) Shrimp TSP3-2 multiple alignment. The nucleotides in the TSP3-2 domains were aligned by the ClustalX software and visualized with the GeneDoc program.

proteins. The shrimp TSP3-2 gene was cloned in frame with a histidine fusion system into the pCold vector (Takara, Tokyo, Japan) and expressed in E. coli BL21(DE3). The His-TF and His-TF-TSP3-2 were examined by SDS-PAGE; they were produced after induction with IPTG as shown in lanes 2 and
4 of Fig. 2a. The His-TF-TSP3-2 protein after purified by affinity chromatography was separated on $12 \%$ SDS-PAGE (Coomassie blue staining); a single band was observed (Fig. 2b). His-TF-TSP3-2 was expected to contain 613 aa, consisting of 459 aa of His-TF and 154 aa of TSP3-2, with a molecular 


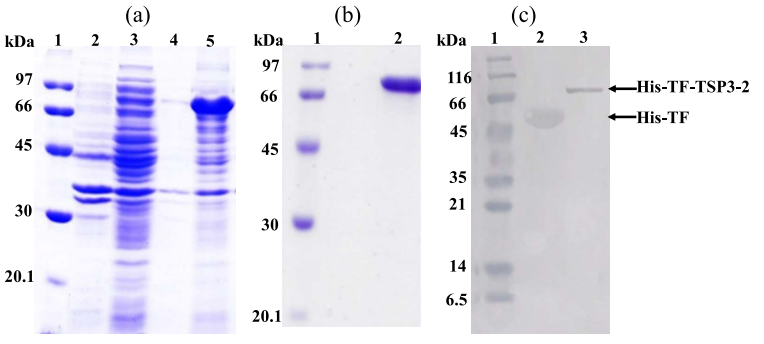

Fig. 2 TSP3 protein expression, purification and detection. (a) The proteins were separated by $12 \%$ SDSPAGE with Coomassie blue staining of the cell-free extracts: lane 1, molecular weight markers; lane 2, noninduced pColdTF cell lysate; lane 3 , induced pColdTF cell lysate; lane 4, non-induced His-TF-TSP3-2 cell lysate; and lane 5, induced His-TF-TSP3-2 cell lysate. (b) Analysis of the purified His-TF-TSP3-2 protein by $12 \%$ SDS-PAGE: lane 1 molecular weight markers and lane 2 the purified protein. (c) His-TF-TSP3-2 was detected with an antiHis monoclonal antibody after Western blotting: lane 1, molecular weight markers; lane 2, His-TF after induction; and lane 3, His-TF-TSP3-2 after induction.

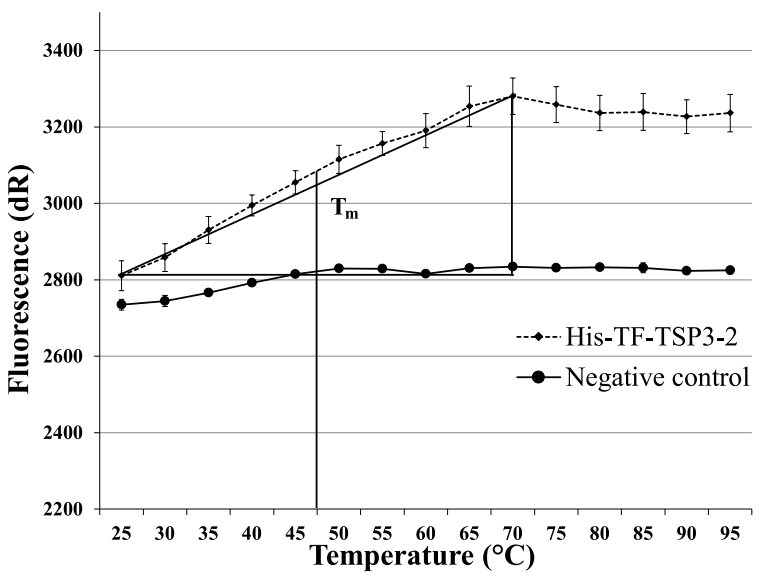

Fig. 3 Thermal stability of His-TF-TSP3-2. The linear line represents the $\mathrm{T}_{\mathrm{m}}$ of His-TF-TSP3-2. Error bars are the mean $\pm \mathrm{SD}(N=3)$.

weight of $69 \mathrm{kDa}$ (Fig. 2b). The His-TF and HisTF-TSP3-2 proteins were detected with an anti-His monoclonal antibody after Western blotting and showed a positive band in lanes 2 and 3, respectively (Fig. 2c).

\section{Biological and physical properties of HIS-TF-TSP3-2}

A thermal shift assay was used to determine the thermal stability of His-TF-TSP3-2. A graph of the relationship between temperature and the fluores-

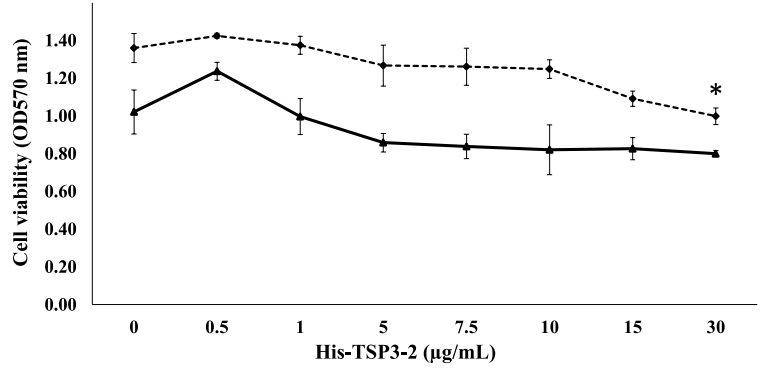

Fig. 4 Cell toxicity of His-TF-TSP3-2 on fibroblast cell lines over $24 \mathrm{~h}$ (solid) and $48 \mathrm{~h}$ (dash) time periods using the MTT assay. Error bars are the mean \pm SD and * indicates a significant difference ( $p<0.05, N=3)$.

cent signal was plotted and analyzed. SYPRO orange can bind to unfolded protein, and aggregation of protein:dye complexes leads to quenching of the fluorescence signal. The melting temperature was identified at the midpoint of the unfolding transition. The assay showed that the melting temperature of His-TF-TSP3-2 was $47.5^{\circ} \mathrm{C}$, which means that the His-TF-TSP3-2 protein could exhibit some activity at room temperature (Fig. 3).

Cytotoxicity was evaluated by the method described [23]. His- TF-TSP3-2 at various concentrations (i.e., $0,0.5,1,5,7.5,10,15$, and $30 \mu \mathrm{g} / \mathrm{mL}$ ) in fresh medium was added to the skin fibroblast cell line. The percentage of cell proliferation was calculated and compared to a negative control after 24 or $48 \mathrm{~h}$ of incubation. Fig. 4 shows that HisTF-TSP3-2 at $0.5 \mu \mathrm{g} / \mathrm{mL}$ increased cell proliferation whereas the other concentrations did not show activity after $24 \mathrm{~h}$ of the treatment of skin fibroblasts. After $48 \mathrm{~h}$ of the treatment with 0.5, 1, 5, 7.5, 10 , or $15 \mu \mathrm{g} / \mathrm{mL}$ His-TF-TSP3-2, the proliferation of the skin fibroblasts was 1.2-1.4 fold increased compared to the control without treatment. Therefore, the best concentration for cell proliferation was $0.5 \mu \mathrm{g} / \mathrm{mL}$.

In addition, the activation of the wound healing of human skin after cell injury by His-TF-TSP3-2 was determined by measuring the migration of a cell population on a scratched surface of the cell monolayer [24]. Cells treated with His-TF-TSP3-2 at a concentration of $0.5 \mu \mathrm{g} / \mathrm{mL}$ showed percent migration rates of $24.00,43.79$, and $67.86 \%$ after treatment for 24,48 , and $72 \mathrm{~h}$, respectively. The percent migration rates of the first control cells (no treatment) were $15.69,27.39$, and $55.84 \%$ at 24 , 48 , and $72 \mathrm{~h}$, respectively. The percent migration rates of the second control cells (His-TF treatment) 


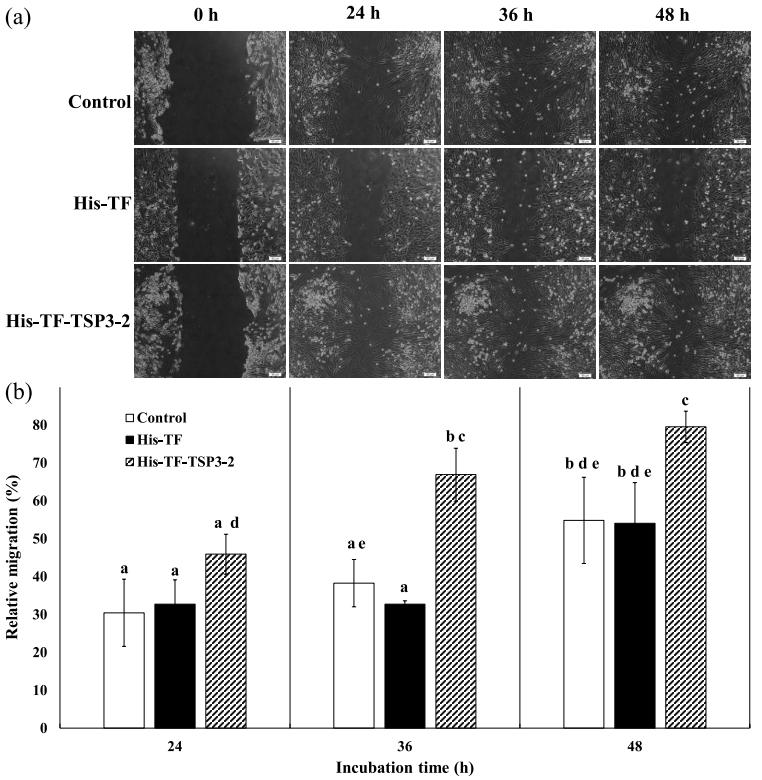

Fig. 5 In vitro scratch assay. (a) Three groups of fibroblast cells were scratched. (b) Percentage of fibroblast cell migration after scratching at 24,36 , and $48 \mathrm{~h}$.

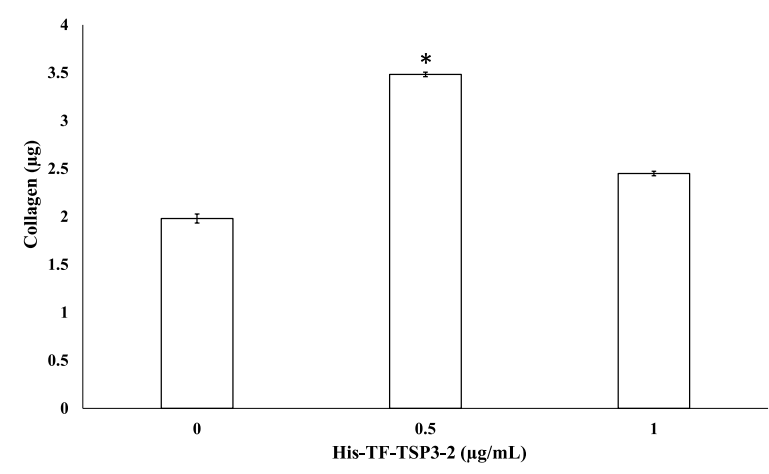

Fig. 6 Collagen assay after treatment of a fibroblast cell line with His-TF-TSP3-2. Error bars are the mean \pm SD and * indicates a significant difference $(p<0.05, N=3)$.

were $21.76,37.56$, and $44.01 \%$ at 24,48 , and $72 \mathrm{~h}$, respectively (Fig. 5). Comparison of the percent migration rates of the His-TF-TSP3-2-treated cells from $0-24,36$, and $48 \mathrm{~h}$ with both controls showed that the percent migration rate of the $0.5 \mu \mathrm{g} / \mathrm{mL}$ His-TF-TSP3-2 group was much faster than those of the controls.

Soluble collagen was also measured in the culture medium of the human skin fibroblast cell line after treatment with His-TF-TSP3-2 for $24 \mathrm{~h}$. The collagen production was 1.7 -fold and 1.2 -fold increased compared with the control after treatment with His-TF-TSP3-2 at concentrations of 0.5 and

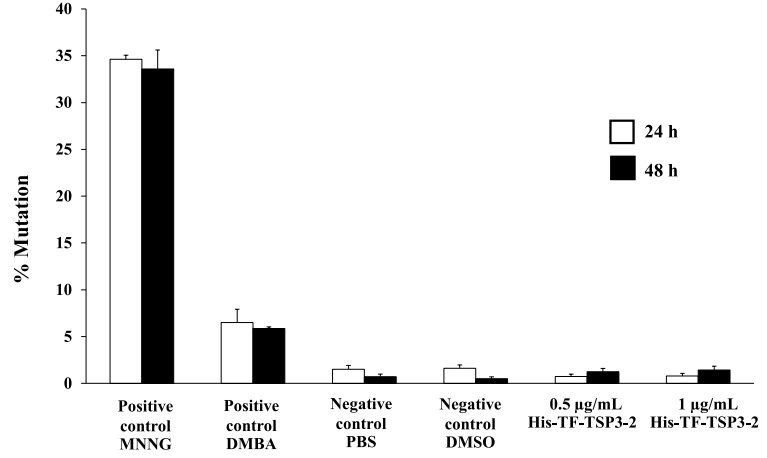

Fig. 7 Mutation assay after treatment of $\mathrm{CHO}$ cells with His-TSP3-2. Error bars indicate the mean \pm SD $(N=3)$.

$1 \mu \mathrm{g} / \mathrm{mL}$, respectively (Fig. 6).

\section{Genotoxicity of the HIS-TF-TSP3-2 protein}

After CHO cells were exposed to 0.5 or $1 \mu \mathrm{g} / \mathrm{mL}$ of His-TF-TSP3-2 protein at $37^{\circ} \mathrm{C}$ for 24,48 , or $72 \mathrm{~h}$, the genotoxicity was evaluated; the MNNG and DMBA mutagens served as positive controls, and the PBS and DMSO were used as negative controls. Mutant cells were selected to determine the mutant frequency, and no significant mutant frequency was found in the His-TF-TSP3-2-treated cells compared to the untreated cells (Fig. 7). The mutant frequencies were $35 \%$ and $6.5 \%$ in the MNNG and DMBA mutagen-treated cells, respectively.

\section{DISCUSSION}

Human EGF is known for its cell proliferation activity in medical applications including wound healing and has been used since 1989 [20]. It has been shown to enhance the healing process in peripheral tissue wounds and gastrointestinal damage. A study in transgenic mice overexpressing EGF showed that this protein may not be sufficient to transform cells, and no effects of EGF were observed on either in vitro or in vivo human gastric cancer cell growth $[25,26]$. In contrast, long-term EGF administration caused epithelial organ hyperplasia in a dose-dependent manner with no change to cell phenotype differentiation, or no tumor markers were found [27]. Clinically, a topical recombinant human EGF was tested in 77 patients with skin lesions and showed promising results; however, the researchers suggested that patients should have individualized formulations for efficient treatment, and large scale studies should be performed [28]. Therefore, the balance between the advantages and disadvantages of human EGF are currently under investigation. 
Thus, an EGF-like domain from other sources should be evaluated to get good candidates for treatment.

The TSP3 proteins from the shrimp species P. monodon, F. chinensis, and F. merguiensis (examined in this study) contained an EGF-like domain whereas 6 and 3 EGF domains have been reported in P. japonicas and human TSP3, respectively. TSP in shrimp has been suggested to act as a protective protein in oocytes, as it is present in the cortical rod in the ovary along with SOP (shrimp ovarian peritrophin). Additionally, TSP is similar to SOP because the structure contains a chitin-binding domain that plays a role in protecting the ovary from microorganism invaders $[29,30]$. However, TSP3 in shrimp was found to increase during the ovarian maturation process, which implies a role of TSP in proliferation activity in the shrimp ovary. The effect of the EGF domain on the proliferation activity of cells was shown in both the MTT and scratch assays in this study. It was also found that treatment with shrimp EGF (His-TF-TSP3-2) at $0.5 \mu \mathrm{g} / \mathrm{mL}$ resulted in 1.7-fold increase in the production of soluble collagen. The role of EGF is also critical at the initial stages of wound healing.

EGF stimulates the proliferation of fibroblasts and keratinocytes around lesions that occur during the early phase of healing [31]. This growth factor has been applied to assist in wound healing. It has been reported that an effect of EGF is to induce the expression of the gene encoding phosphatidylinositol 3-kinase (PI 3-kinase) during rabbit corneal epithelial wound repair [32]. The stimulation of cell line migration by commercial EGF has been reported. EGF induces cell migration in cultured human lens epithelial cells through the ERK and PI3k/AKT pathways [15]. Therefore, the ability of our recombinant EGF (His-TF-TSP3-2) to contribute to cell migration indicated that this EGF protein has a similar function to commercial EGF. Since only $30 \%$ nucleotide sequence similarity between EGF from FmTSP3 and human TSP3 was observed, shrimp EGF may present some differences from that of humans in terms of activity and specificity. Therefore, EGF (His-TF-TSP3-2) from FmTSP was evaluated for genotoxicity, and no significant mutant frequency was found in His-TF-TSP3-2-treated cells compared to untreated cells.

Moreover, our recombinant EGF protein increased the collagen level of the skin fibroblast cell lines. Several studies have shown that EGF affects collagen biosynthesis in cultured cells [19]. EGF increases the contents of acidic glycosaminoglycans (AGAGs), which are thought to be closely related to the formation of collagen fibers [33]. AIMP1derived peptide (AdP, INCI name: sh-oligopeptide5/sh-oligopeptide SP) has been proposed as an alternative product to EGF because of its high sensitivity to the EGF concentrations applied [34]. Although the recombinant His-TF-TSP3-2 had quite a high melting temperature and low sensitivity in humans, further pharmaceutical testing of this TSP3-2 protein is required to confirm its benefit as an antiwrinkle or a skin renewal agent.

Acknowledgements: We would like to acknowledge the financial supports from the Center of Excellence on Medical Biotechnology (CEMB), the S\&T Postgraduate Education and Research Development Office (PERDO), the Office of the Higher Education Commission (OHEC), and the Thai Government Supracluster Project, contract no. SCI600739S, Thailand.

\section{REFERENCES}

1. Bornstein P (2001) Thrombospondins as matricellular modulators of cell function. J Clin Invest 107, 929-934.

2. Stenina-Adognravi O (2014) Invoking the power of thrombospondins: Regulation of thrombospondins expression. Matrix Biol 37, 69-82.

3. Lawler PR, Lawler J (2012) Molecular basis for the regulation of. Cold Spring Harb Perspect Med 2, ID a006627.

4. Martin-Manso G, Navarathna DHMLP, Galli S, SotoPantoja DR, Kuznetsova SA, Tsokos M, Roberts DD (2012) Endogenous thrombospondin-1 regulates leukocyte recruitment and activation and accelerates death from systemic candidiasis. PLoS One 7, ID e48775.

5. Sweetwyne MT, Murphy-Ullrich JE (2012) Thrombospondin 1 in tissue repair and fibrosis: TGF- $\beta$ dependent and independent mechanisms. Matrix Biol 31, 178-186.

6. Adams JC, Lawler J (2011) The thrombospondins. Cold Spring Harb Perspect Biol 3, ID a009712.

7. Yamano K, Qiu G-F, Unuma T (2004) Molecular cloning and ovarian expression profiles of thrombospondin, a major component of cortical rods in mature oocytes of Penaeid shrimp, Marsupenaeus japonicus. Biol Reprod 70, 1670-1678.

8. Zhou F, Zheng L, Zhang D, Huang J, Qiu L, Yang Q, Jiang S (2011) Molecular cloning, characterization and expression analysis of thrombospondin gene from Penaeus monodon. Mar Genomics 4, 121-128.

9. Kaiser R, Grotemeyer K, Kalsch T, Graber S, Wilkens H, Elmas E (2013) Decreased TSP-1 following percutaneous coronary intervention is associated with major adverse cardiac events in ST-elevation myocardial infarction. Clin Hemorheol Microcirc 54, 59-73. 
10. Rosini S, Pugh N, Bonna MA, Hulmes DJS, Farndale WR, Adams JC (2018) Thrombospondin-1 promotes matrix homeostasis by interacting with collagen and lysyl oxidase precursors and collagen cross-linking sites. Sci Signal 11, ID eaar2566.

11. Lynch JM, Maillet M, Vanhoutte D, Schloemer A, Sargent MA (2012) A Thrombospondin-dependent pathway for a protective ER stress response. Cell 149, 1257-1268.

12. Palao T, Medzikovic L, Rippe C, Wanga S, Al-mardini C, Weert A Van, Vos J De, Wel NN Van Der, et al (2018) Thrombospondin-4 mediates cardiovascular remodelling in angiotensin II-induced hypertension. Cardiovasc Pathol 35, 12-19.

13. Chen FH, Thomas AO, Hecht JT, Goldring MB, Lawler J (2005) Cartilage oligomeric matrix protein/thrombospondin 5 supports chondrocyte attachment through interaction with integrins. $J$ Biol Chem 280, 32655-32661.

14. Sun YD, Zhao XF, Kang CJ, Wang JX (2006) Molecular cloning and characterization of Fc-TSP from the Chinese shrimp Fennerpenaeus chinensis. Mol Immunol 43, 1202-1210.

15. Jiang Q, Zhou C, Bi Z, Wan Y (2006) EGF induced cell migration in cultured human lens epithelial cells through the ERK and PI3K/AKT pathways. $J$ Ocul Pharmacol Ther 22, 93-102.

16. Bodnar RJ (2013) Epidermal growth factor and epidermal growth factor receptor: The Yin and Yang in the treatment of cutaneous wounds and cancer. $A d v$ Wound Care 2, 24-29.

17. Brown GL, Nanney LB, Griffen J, Cramer AB, Yancey JM, Curtsinger LJ, Holtzin L, Schultz GS, et al (1989) Enhancement of wound healing by topical treatment with epidermal growth factor. $N$ Engl $J$ Med 321, 76-79.

18. Losada F, García-Luna PP, Gómez-Cía T, Garrido M, Pereira JL, Marín F, Astorga R (2002) Effects of human recombinant growth hormone on donor-site healing in burned adults. World J Surg 26, 2-8.

19. Jia YY, Zhou JY, Chang Y, An F, Li XW, Xu XY, Sun XL, Xiong CY, et al (2018) Effect of optimized concentrations of basic fibroblast growth factor and epidermal growth factor on proliferation of fibroblasts and expression of collagen: related to pelvic floor tissue regeneration. Chin Med J 131, 2089-2096.

20. Choi JS, Leong KW, Yoo HS (2008) In vivo wound healing of diabetic ulcers using electrospun nanofibers immobilized with human epidermal growth factor (EGF). Biomaterials 29, 587-596.

21. Lynch TJ, Kim ES, Eaby B, Garey J, West DP, Lacouture ME (2007) Epidermal growth factor receptor inhibitor-associated cutaneous toxicities: An evolving paradigm in clinical management. Oncologist 12, 610-621.

22. Lo M, Aulabaugh A, Jin G, Cowling R, Bard J, Malamas M, Ellestad G (2004) Evaluation of fluorescence- based thermal shift assays for hit identification in drug discovery. Anal Biochem 332, 153-159.

23. Aramwit P, Kanokpanont S, De-Eknamkul W, Srichana $T$ (2009) Monitoring of inflammatory mediators induced by silk sericin. $J$ Biosci Bioeng 107, 556-561.

24. Fronza M, Heinzmann B, Hamburger M, Laufer S, Merfort I (2009) Determination of the wound healing effect of Calendula extracts using the scratch assay with 3 T3 fibroblasts. $J$ Ethnopharmacol 126, 463-467.

25. Berlanga-Acosta J, Gavilondo-Cowley J, López-Saura P, González-López T, Castro-Santana MD, López-Mola E, Guillén-Nieto G, Herrera-Martinez L (2009) Epidermal growth factor in clinical practice - a review of its biological actions, clinical indications and safety implications. Int Wound $J$ 6, 331-346.

26. Xia L, Yuan YZ, Xu C Di, Zhang YP, Qiao MM, Xu JX (2002) Effects of epidermal growth factor on the growth of human gastric cancer cell and the implanted tumor of nude mice. World J Gastroenterol 8, 455-458.

27. Reeves JR, Richards RC, Cooke T (1991) The effects of intracolonic EGF on mucosal growth and experimental carcinogenesis. Br J Cancer 63, 223-226.

28. Esquirol-Caussa J, Herrero-Vila E (2019) Human recombinant epidermal growth factor in skin lesions: 77 cases in EPItelizando project. $J$ Dermatolog Treat 30, 96-101.

29. Loongyai W, Avarre J-C, Cerutti M, Lubzens E, Chotigeat W (2007) Isolation and functional characterization of a new shrimp ovarian peritrophin with antimicrobial activity from Fenneropenaeus merguiensis. Mar Biotechnol 9, 624-637.

30. Anuchan S, Deachamag P, Siammai N, Phongpaichit S, Chotigeat W (2015) Antimicrobial activity of engineered shrimp ovarian peritrophin fragments from Fenneropenaeus merguiensis. Protein Pept Lett 22, 73-80.

31. Choi JK, Jang J-H, Jang W-H, Kim J, Bae I-H, Bae J, Park Y-H, Kim BJ, et al (2012) The effect of epidermal growth factor (EGF) conjugated with low-molecularweight protamine (LMWP) on wound healing of the skin. Biomaterials 33, 8579-8590.

32. Zhang Y, Liou GI, Gulati AK, Akhtar RA (1999) Expression of phosphatidylinositol 3-kinase during EGF-stimulated wound repair in rabbit corneal epithelium. Investig Visual Sci 40, 2819-2826.

33. Kumegawa M, Hiramatsu M, Yajima T, Hatakeyama K, Hosoda S, Namba M (1982) Effect of epidermal growth factor on collagen formation in liver-derived epithelial clone cells. Endocrinology 110, 607-612.

34. Kim J, Sujin MS, Ms K, Kwon H, Moon H, Park MC (2019) Dual functional bioactive-peptide, AIMP1derived peptide (AdP), for anti-aging. $J$ Cosmet Dermatol 18, 251-257. 